\title{
Epidemiology of Diabetes and Hearing Loss
}

\author{
Rachael R. Baiduc, Ph.D., M.P.H. ${ }^{1}$ and Elizabeth P. Helzner, Ph.D., M.S. ${ }^{2}$
}

ABSTRACT

Hearing loss is a highly prevalent chronic condition. In addition to age, sex, noise exposure, and genetic predisposition, cardiovascular disease and its antecedents may precipitate hearing loss. Of emerging interest is the connection between diabetes and auditory dysfunction. Cross-sectional studies consistently suggest that prevalence of hearing loss is higher in persons with diabetes compared with those without diabetes, especially among younger persons. Furthermore, longitudinal studies have demonstrated higher incidence of hearing loss in persons with diabetes compared to those without diabetes. These findings seem to hold for both type 1 and type 2 diabetes, although considerably more population-based evidence is available for type 2 diabetes. Data on gestational diabetes and hearing outcomes are limited, as are data relating diabetes to otologic sequelae such as fungal infection. Here, we examine evidence from epidemiologic studies of diabetes and hearing loss and consider clinical and laboratory data where population-based data are lacking.

KEYWORDS: hearing loss, diabetes, epidemiology

\section{DIABETES: DEFINITION AND PREVALENCE}

Diabetes mellitus (DM) is a multisystem chronic condition characterized by high blood and urine glucose levels due to inadequate insulin production or utilization. It is a prevalent chronic condition in the United States, with an estimated $9.9 \%$ of the population over age 20 years (i.e., 21 million adults) with confirmed diabetes and an additional $12.4 \%$ with undiagnosed disease. ${ }^{1}$ Individuals in certain racial/ethnic groups may be more at risk. For example, the prevalence of diagnosed diabetes is higher among nonHispanic black individuals compared to nonHispanic whites. ${ }^{2}$ Furthermore, among older adults ( $\geq 65$ years), an estimated $45.6 \%$ of diabetes cases are undiagnosed and the prevalence of diabetes is more than two times higher in elderly persons compared to those of young or middle age. ${ }^{2}$ The estimated worldwide prevalence of DM (type 1 and 2) is $6.4 \%$, with a predicted prevalence of $7.7 \%$ by $2030 .^{3}$

\footnotetext{
${ }^{1}$ Department of Speech, Language, and Hearing Sciences, University of Colorado Boulder, Boulder, Colorado; ${ }^{2}$ Department of Epidemiology and Biostatistics, School of Public Health, SUNY Downstate Medical Center, Brooklyn, New York.

Address for correspondence: Rachael R. Baiduc, Ph.D., M.P.H., Department of Speech, Language, and Hearing Sciences, University of Colorado Boulder, 2501 Kittredge

Loop, 409 UCB, Boulder, CO 80309-0409

(e-mail: Rachael.Baiduc@colorado.edu).

The Role of Audiology in the Care of Persons with Diabetes; Guest Editor, Victor Bray, Ph.D.

Semin Hear 2019;40:281-291. Copyright (C) 2019 by Thieme Medical Publishers, Inc., 333 Seventh Avenue, New York, NY 10001, USA. Tel: +1(212) 584-4662.

DOI: https://doi.org/10.1055/s-0039-1697643.
} 
DM, characterized by hyperglycemia, is a group of metabolic diseases. There are two types of DM: type $1 \mathrm{DM}$ (T1DM) results from absolute deficiency of insulin secretion as a result of $\beta$-cell destruction and accounts for less than $10 \%$ of diabetes cases. ${ }^{4} \mathrm{~T} 1 \mathrm{DM}$ is the third most common chronic disease in childhood ${ }^{5}$ and is estimated to affect approximately 1 in 433 individuals younger than 20 years in the United States. ${ }^{6}$ Although often termed "juvenile-onset diabetes," recent epidemiological reports suggest the incidence is now comparable in children and adults, signifying the disease may develop across the lifespan. ${ }^{7}$ It is predicted that increases in incidence, and therefore prevalence, of T1DM will primarily occur among individuals in minority racial/ethnic groups. ${ }^{8}$ In contrast, type $2 \mathrm{DM}$ (T2DM) results from progressive loss of $\beta$-cell insulin secretion and inadequate insulin secretion response and accounts for approximately 90 to $95 \%$ of DM cases. ${ }^{9}$ It is often diagnosed later in life relative to T1DM. There are an estimated 30.3 million persons with diabetes (PWD) in the United States, or 9.4\% of the population. The prevalence of diabetes increases markedly with age, with $25.2 \%$ of those aged 65 years or older affected. T2DM is much more common than T1DM, and accounts for approximately $95 \%$ of diabetes cases. ${ }^{10}$

Diabetes has severe consequences; it was the third leading cause of death in the United States, accounting for $11.7 \%$ of deaths, based on data from the National Health and Nutrition Examination Survey (NHANES) ${ }^{11}$ and is associated with numerous comorbidities. Diabetes-associated micro- and macrovascular compromise can cause retinopathy, peripheral neuropathy, and nephropathy (among other complications), ${ }^{12}$ leading to clear clinical guidelines by the American Diabetes Association (ADA) that recommend examination of these issues during a comprehensive medical evaluation. ${ }^{13}$ However, the extensive medical evaluation currently suggested by the ADA does not yield identification of all sequelae of DM. Many PWD also suffer from sensorineural hearing loss (SNHL), and although hearing loss $i s$ mentioned as a possible consequence of DM in the most recent medical evaluation of comorbidities published by the $\mathrm{ADA},{ }^{13}$ a hearing evaluation is not included in the advocated end-organ assessment.
Auditory compromise was first considered as a consequence of DM in $1857 .{ }^{14}$ Since then, much work has been done in this area although auditory dysfunction remains an underrecognized complication of DM and recommendations for hearing loss screening in this population are currently lacking. Here we provide evidence from population-based studies and meta-analyses that support and refute the connection between DM and hearing loss. We also include brief discussions of laboratory and clinical data, particularly where population-based data are unavailable (i.e., for T1DM). We consider T1DM and T2DM separately where possible and review the limited data on gestational diabetes and auditory health. We seek to answer the question "Is DM-related auditory dysfunction a variant of age-related hearing loss, secondary to other DM-related comorbid conditions (obesity, cardiovascular diseases, hypertension, etc.) or a unique pathological condition we deem the diabetic ear?"

\section{T1DM AND HEARING LOSS}

Most research of DM and auditory dysfunction has focused on T2DM. Nonetheless, there are several smaller laboratory or clinical studies that have evaluated the relationship between T1DM and SNHL. Owing to the small sample sizes in these studies, reliable prevalence estimates of SNHL in persons with T1DM are unavailable. What follows is a summary of results available from clinical and laboratory studies.

Malucelli et al compared hearing thresholds from 250 to $16,000 \mathrm{~Hz}$ in 30 individuals with T1DM (mean age $=25.9$ years) to 30 controls (mean age $=26.56$ years). Both groups were $57 \%$ male. They identified significantly poorer low$(250$ and $500 \mathrm{~Hz})$ and high-frequency $(10,000$, $11,200,12,500,14,000$, and $16,000 \mathrm{~Hz}$ ) hearing thresholds in PWD compared with controls. The observed between-group differences in this study were large, up to nearly $20 \mathrm{~dB}$ at $16,000 \mathrm{~Hz} .{ }^{15}$

A study of 63 patients ( $<18$ years) and 63 age- and sex-matched controls who underwent pure-tone audiometry showed that PWD had poorer hearing from 2,000 to $8,000 \mathrm{~Hz} .{ }^{16}$ The observed elevation in behavioral thresholds was higher among those with longer disease duration and/or poorer metabolic control. Another case- 
control study evaluated 100 young PWD and 100 controls (aged 5-18 years) and found significantly elevated behavioral pure-tone thresholds from 250 to $8,000 \mathrm{~Hz}$, although the observed mean thresholds of the diabetic group would place the degree of loss as slight. ${ }^{17}$ Moreover, individuals with longer duration of diabetes $(\geq 5$ vs. $<5$ years) had significantly elevated thresholds across the frequency range.

A separate evaluation of 50 cases (mean age: 25.6 years) and 50 controls (mean age $=27.6$ years) also demonstrated elevated behavioral thresholds at most frequencies from 250 to $8,000 \mathrm{~Hz}$ (excluding $500 \mathrm{~Hz}$ in the right ear and $2,000 \mathrm{~Hz}$ in the left ear) in young PWD compared with controls. ${ }^{18}$ Differences were most evident at $8,000 \mathrm{~Hz}$. Last, a small investigation of 13 newly diagnosed patients and 33 with T1DM for $3(+)$ years found significantly elevated thresholds from 250 to $8,000 \mathrm{~Hz}$ compared with age-matched controls. ${ }^{19}$

In contrast, Botelho et $\mathrm{al}^{20}$ did not observe significant differences in behavioral thresholds of young PWD (mean age $=14.12$ years, $n=40)$ compared with age- and sex-matched controls $(n=40)$ but did detect lower distortion product otoacoustic emission (DPOAE) amplitudes at 6,000 and $7,969 \mathrm{~Hz}$ in diabetics compared with nondiabetics. In a related study, the OAEs of 60 PWD (mean age $=31$ years, \pm 6.23 years) were compared with 58 controls. ${ }^{21}$ All participants in their study demonstrated clinically normal hearing with no significant between-group differences in hearing thresholds. Significantly reduced (poorer) DPOAE amplitudes were observed across the frequency range from 949 to $3,626 \mathrm{~Hz}$ in the PWD group. Lisowska et $\mathrm{al}^{22}$ compared auditory profiles of 42 patients with T1DM to 33 age- and sex-matched controls. The PWD had clinically normal hearing and DPOAEs were significantly reduced in amplitude in the PWD compared with controls from 1,000 to $6,000 \mathrm{~Hz}$. Hou et al's ${ }^{18}$ study demonstrated significantly lower (poorer) DPOAE amplitudes at $4,000,6,000$, and $8,000 \mathrm{~Hz}$ in young (mean age $=25.6$ years) diabetics compared with 50 age- and sex-matched controls (mean age $=27.6$ years). These data hint at the possibility of diabetic cochleopathy, even in the presence of clinically normal hearing.
These reports and others were recently evaluated in a meta-analysis of 15 clinical studies. The studies included a range of pure-tone audiometry, OAE, and auditory brainstem response (ABR) data. Prevalence estimates were synthesized across four studies for a total of 252 diabetic cases and 253 controls. The pooled odds ratio of hearing loss in these cases was 49.08 (95\% confidence interval [CI], 12.03-200.31). These results may be interpreted with some caution given that the largest sample size in any of the four studies was 100 PWD and 100 controls. Moreover, data from only four studies (specifically 252 cases [PWD] and 253 controls) were considered for calculation of this pooled prevalence estimate. Combining results across six studies, significantly poorer behavioral thresholds were observed only at $4,000 \mathrm{~Hz}$. Note that the auditory dysfunction observed in these studies was typically subclinical. ${ }^{23}$

These smaller, case-control studies of T1DM further substantiate the association between DM and hearing loss and add to the considerable body of work on hearing loss and T2DM. Most of the studies of T1DM and hearing have been relatively small laboratorybased designs and to our knowledge, there are no large, population-based reports examining the association between T1DM and SNHL; hence, incidence data and precise populationbased prevalence estimates are not readily available. As most of the T1DM studies have evaluated hearing status in younger persons, these data support the argument that diabetesrelated changes to the auditory system are separate from overall auditory aging. Furthermore, the work of Hou et a ${ }^{18}$ excluded individuals with occupational noise exposure, ruling out acoustic trauma as a likely cause of hearing loss.

\section{DIABETES MELLITUS AND HEARING LOSS: PREVALENCE DATA}

In general, epidemiological data support an association between DM and hearing loss. Most population-based studies do not differentiate between T1DM and T2DM; hence, the data discussed here are nonspecific with regard to diabetes type unless otherwise specified. We 
first present cross-sectional (prevalence) data and then evaluate longitudinal (causal) data.

Population-based research has evaluated the association between diabetes and prevalent hearing loss using data from the NHANES; the Beaver Dam Study; the Health, Aging, and Body Composition (Health ABC) study; the Epidemiology of Hearing Loss Study (EHLS); the Australian Blue Mountains Hearing Study; and the Hispanic Community Health Study/ Study of Latinos (HCHS/SOL).

Multiple studies examining the association between prevalent hearing loss and T2DM have been performed in the NHANES, an ongoing population-based study of noninstitutionalized persons designed to be representative of the $U$. S. population. ${ }^{24}$ A 2009 report explored trends in the prevalence of hearing loss in the United States in persons aged 25 to 69 years with and without diabetes. Data from the 1971-1973 and 1999-2004 cycles were considered. This study found that the prevalence of hearing loss in persons without diabetes declined over time (from 24.4\% [95\% CI, 22.3-26.6\%] to $22.3 \%$ [95\% CI, 20.4-24.2]), whereas the prevalence of hearing loss in PWD increased (from 28.5\% [95\% CI, 20.4-36.6\%] to 34.4\% [95\% CI, 29.1$39.7 \%]$ ). Prevalence estimates were adjusted for age, sex, race, and education level. These results suggest that not only do PWD have a higher prevalence of hearing loss, they have not had a reduction in hearing loss since the 1970s as have persons without diabetes. ${ }^{25}$

Diabetes was associated with greater odds of low- and high-frequency hearing loss (odds ratio $[\mathrm{OR}]=2.03[95 \% \mathrm{CI}, 1.32-3.10]$ and $\mathrm{OR}=1.67$ [95\% CI, 1.14-2.44], respectively) in an evaluation of 1,508 NHANES participants from the 1999-2004 cycle. Participants were aged 40 to 69 years and estimates were adjusted for demographic characteristics and noise exposure. ${ }^{26}$ In a related study, 536 PWD (aged 20-69) from the 1999-2004 NHANES cycle underwent pure-tone audiometry (from 500 to $8,000 \mathrm{~Hz}$ ). The goal of this study was to assess the relationship between cardiovascular disease risk factors and prevalent hearing loss among PWD. The role of glycemic control also was considered. The weighted prevalence of low-frequency hearing loss in this population (defined as average threshold at $500,1,000$, and $2,000 \mathrm{~Hz}$ ) was $34.6 \%$, while high-frequency hearing loss (average threshold at $3,000,4,000,6,000$, and $8,000 \mathrm{~Hz}$ ) was more common at $65.5 \%$. Years since diabetes diagnosis and suboptimal glycemic control were not significantly associated with low- or high-frequency hearing loss. Among these PWD, individuals with coronary heart disease had increased odds of high-frequency hearing loss compared with PWD without coronary heart disease $\left(\mathrm{OR}=4.39\right.$ [95\% CI, 1.26-15.26]). ${ }^{27}$

Other population-based data support the association between hearing loss and diabetes. For example, in an analysis of 3,571 participants from the Beaver Dam study, persons with T2DM were more likely than those without diabetes to have prevalent hearing loss (59 vs. $44 \%$ ), although the difference was not statistically significant when controlled for age. ${ }^{28} \mathrm{Hear}-$ ing loss was defined as pure-tone average (at 500, $1,000,2,000$, and $4,000 \mathrm{~Hz}$ ) greater than $25 \mathrm{~dB}$ HL. However, once individuals with presbycusis-like audiometric phenotypes were excluded from analysis, a significant association between T2DM and hearing loss was observed (OR $=1.41$ [95\% CI, 1.05-1.88, $p=0.02]$ ).

A random sample of Medicare beneficiaries enrolled in the Health ABC study (73-84 years [mean: 77.5 years]; $n=2,049)$ were evaluated in terms of prevalent hearing loss and diabetes status. Data were stratified by race (black or white) and sex. After stratification, diabetes was associated with prevalent hearing loss (defined as pure-tone average 500, 1,000, and 2,000 Hz $>25 \mathrm{~dB} \mathrm{HL}$ ) in white men and women. After adjustment for potential confounders, compared with those without diabetes, the odds of hearing loss among white men with diabetes were 2.12 (95\% CI, 1.17-4.49) and among white women with diabetes, the odds of hearing loss were 1.89 (95\% CI, 1.07-3.35). In multivariable-adjusted models, diabetes also was associated with highfrequency hearing loss (average threshold at 2,000, 4,000, and $8,000 \mathrm{~Hz}>40 \mathrm{~dB} \mathrm{HL}$ ), but only in black women. ${ }^{29}$

The HCHS/SOL evaluated the association between prevalent SNHL (as indicated by PTA $[500,1000,2000$, and $4000 \mathrm{~Hz}]>25 \mathrm{~dB} \mathrm{HL}$ in either ear) and diabetes in 16,415 Hispanic/ Latino(a) adults aged 18 to 74 years. The adjusted (age, sex, center, and Hispanic/Latino 
background) odds of bilateral hearing loss was significantly higher in PWD than those without $\left(\mathrm{OR}=1.20\right.$ [95\% CI, 1.21-2.08]). ${ }^{30}$

Associations between DM and hearing loss also have been found in large studies of veterans. Kakarlapudi et $\mathrm{al}^{31}$ retrospectively examined electronic medical records of 12,575 PWD and 53,461 age-matched nondiabetics from the VA Maryland Health Care system. SNHL was identified via ICD codes. The prevalence of hearing loss was $13.1 \%$ in PWD compared with $10.3 \%$ in the nondiabetic group $(p<0.05)$. For a subset of 1,888 PWD and SNHL, audiometric data were available. Higher (poorer) hearing thresholds and lower (poorer) speech discrimination scores correlated with increasing creatinine levels. Similarly, Austin et $\mathrm{al}^{32}$ performed a smaller study of behavioral thresholds from 250 to $14,000 \mathrm{~Hz}$ in 302 veterans classified as T1DM, T2DM, or nondiabetic. Three age groups $(<50,50-56$, and $>57$ years) were considered. Among the youngest subjects ( $<50$ years), thresholds were higher (poorer) in persons with T2DM compared to those without across the frequency range. For young persons with T1DM, significant differences were observed only for frequencies less than $2,000 \mathrm{~Hz}$ and above $8,000 \mathrm{~Hz}$. For middle aged and older participants, low-frequency (but not high-frequency) differences in behavioral thresholds were observed.

Finally, there is the possibility that prediabetes might be associated with increased risk of hearing loss as suggested by a recent study of participants from the Korean NHANES. Persons $\geq 20$ years $(n=4,085)$ underwent puretone audiometry from 500 to $6,000 \mathrm{~Hz}$ with prevalent hearing loss defined as pure-tone average $(500,1,000,2,000 \mathrm{~Hz})>25 \mathrm{~dB} \mathrm{HL}$ in the better ear. Those with impaired fasting glucose (i.e., glucose levels $>5.5$ to $\geq 7.0$ $\mathrm{mmol} / \mathrm{L}$ ) had a higher prevalence of hearing loss than those with normal glucose levels (42.2 vs. $24.5 \%)$. For men younger than 70 years, impaired fasting glucose remained an independent risk factor for high-frequency $(3,000$, 4,000 , and $6,000 \mathrm{~Hz}$ ) mild hearing loss in multivariable-adjusted models. Data from the HCHS/SOL also identified a significant association between prediabetes and audiometrically identified bilateral hearing loss $(\mathrm{OR}=1.44$ [95\% CI, 1.07-1.94]). ${ }^{30}$

\section{STUDIES OUTSIDE THE UNITED STATES}

The relationship between DM and SNHL has been assessed outside of the United States. The Australian Blue Mountains Hearing Study (BMHS) is a population-based survey of presbycusis conducted from 1997 to 2004. Multiple hearing measurements were taken (baseline, 5-year follow-up [1997-1999], and again at the 10-year follow-up [2002-2004]). Hearing loss was defined as PTA $(500,1,000,2,000$, and $4,000 \mathrm{~Hz}$ ) greater than $25 \mathrm{~dB}$ HL in the betterhearing ear. Age-adjusted thresholds were poorer in PWD from 250 to $8,000 \mathrm{~Hz}$ (all $p<0.05$ ). In a multivariable model (adjusting for age, sex, education, smoking, and occupational noise exposure), PWD were 50\% more likely to have bilateral hearing loss than those without diabetes $(\mathrm{OR}=1.55$ [95\% CI, 1.11-2.17]). Furthermore, hearing loss progressed over time in the majority (69.6\%) of persons with newly diagnosed diabetes. After multivariable adjustment, compared with those without diabetes, persons with newly diagnosed diabetes were almost three times as likely to experience a decline in hearing sensitivity over time $(\mathrm{OR}=2.71$ [95\% CI, 1.07-6.86]). These data suggest increased prevalence and/or earlier onset of SNHL in PWD compared with those without diabetes. ${ }^{33}$

Uchida et al evaluated the association between prevalent hearing loss and DM in a study of 2,306 adults aged 40 to 86 years from the Longitudinal Study of Aging in Japan. Behavioral thresholds from 125 to $8,000 \mathrm{~Hz}$ were considered and hearing loss was defined as PTA (low, 125, 250, and $500 \mathrm{~Hz}$ or high, 2,000, 4,000 , and $8,000 \mathrm{~Hz}$ ) greater than $25 \mathrm{~dB} \mathrm{HL}$. When considering the poorer hearing ear, the prevalence of hearing loss was higher in PWD than in persons without diabetes at low and high frequencies for individuals aged 40 to 64 years (low, 31.3 vs. $19.2 \%$ and high, 59.7 vs. $29.0 \%$ [both $p<0.05]$ ). For participants aged 65 to 86 years, no significant differences in prevalence of hearing loss were observed based on diabetes status. ${ }^{34}$

The association between prevalent hearing loss and diabetes also was evaluated in male middle-aged self-defense personnel in Japan. Hearing loss was defined as PTA $(500,1,000$, 2,000 , and $4,000 \mathrm{~Hz}$ ) greater than $25 \mathrm{~dB}$ in the 
worse ear. High-frequency hearing loss (average threshold at 3,000, 4,000, and $6,000 \mathrm{~Hz}$ ) also was evaluated. Subjects were divided into three groups: nondiabetic $(n=442)$, impaired glucose tolerance $(n=154)$, and identified DM $(n=103)$. In age-adjusted models, T2DM was significantly associated with prevalent hearing loss $\quad(\mathrm{OR}=1.87 \quad[95 \% \quad \mathrm{CI}, \quad 1.20-2.91])$. Impaired glucose tolerance was not associated with significantly elevated odds of hearing loss $(\mathrm{OR}=1.16$ [95\% CI, 0.80-1.68]). Significant threshold elevation was observed at 1,000, 3,000, and $8,000 \mathrm{~Hz}$ in PWD compared with the nondiabetic group. Differences between the PWD persons with normal glucose tolerance were approximately $2 \mathrm{~dB}$ for low frequencies and $4 \mathrm{~dB}$ for high frequencies. ${ }^{35}$

Last, the Brazilian Longitudinal Study of Adult Health (ELSA-Brasil) examined hearing outcomes in 901 adults with (mean age: 57.4 years, $+/-9.0$ years; $n=191$ ) and without (51.2 years $+/-8.9, n=710)$ diabetes. Although diabetes was associated with low-mid frequency hearing loss $(250-2,000 \mathrm{~Hz})$ in PWD, the significance of this finding did not persist in age, sex, and hypertension-adjusted models (OR $=1.03$ [95\% CI, 0.56-1.92]). The same held true for high-frequency hearing loss $(3,000-8,000 \mathrm{~Hz}){ }^{36}$

\section{META-ANALYSES}

A recent meta-analysis of 18 studies focused exclusively on studies of T2DM and hearing outcomes including behavioral thresholds and ABRs. The majority of these studies were smaller laboratory-based reports (with the exception of the study of Dalton et $\mathrm{al}^{28} \mathrm{a}$ population-based study of individuals from the Beaver Dam study). Hearing loss was defined by all studies as PTA greater than $25 \mathrm{~dB}$ HL in the poorer ear. ABRs were considered in terms of wave I, III, and V latencies. Six of the studies provided prevalence measures, with four of the six demonstrating significantly higher prevalence of hearing loss in PWD compared with persons without. However, some of the included studies were limited in sample size, with less than 100 subjects per group (e.g., Mozaffari et $\left.\mathrm{al}^{37}\right)$. The pooled odds of hearing loss were higher in PWD $(\mathrm{OR}=1.91$ [95\% CI, 1.47-
2.49]). This finding held for younger $(<65$ years) and older subgroups. Furthermore, five of seven studies identified significantly poorer behavioral hearing thresholds in PWD compared with persons without diabetes. In general, the studies included in this meta-analysis support the observation of poorer hearing in diabetics from 500 to $8,000 \mathrm{~Hz}$, with the most pronounced effects at high frequencies $(6,000$ $8,000 \mathrm{~Hz}$ ). Some of the studies included also hint at central auditory dysfunction, as evidenced by significantly longer ABR latencies for waves I, III, and V with the strongest support for increases in wave $\mathrm{V}$ latency. ${ }^{38}$

A second meta-analysis included crosssectional studies of hearing loss and T1DM and T2DM. Data from 13 studies (20,194 persons [7,377 PWD]) were evaluated. Analysis included both population-based and smaller hospital-based studies. The pooled odds of hearing loss across all 13 studies was higher in PWD compared with those without (OR $=2.15$ [95\% CI, 1.72-2.68]). Data also were stratified by age, sex, and other demographic characteristics. The stratified analyses suggested that the significant association between $\mathrm{DM}$ and hearing loss was maintained for young $(<60$ years) and older ( $>60$ years) persons, although there was a stronger association for younger participants than older participants $(\mathrm{OR}=2.61[95 \% \mathrm{CI}, 2.00-3.45] \mathrm{vs}, \mathrm{OR}=1.58$ [95\% CI, 1.38-1.81], respectively). ${ }^{39}$

\section{DIABETES MELLITUS AND HEARING LOSS: INCIDENCE DATA}

One of the key questions left unanswered by the above cross-sectional data is that of causation. Longitudinal studies are helpful in this regard, as the temporal relationship between diabetes and incidence of hearing loss can be examined. Although there are fewer incidence studies than prevalence (cross-sectional) studies, they generally support modestly increased risk of incident hearing loss in PWD compared with persons without diabetes.

One example comes from the Beaver Dam Study. Participants in the Beaver Dam Eye Study were invited to participate in an ancillary study, the Epidemiology of Hearing Loss Study (EHLS). Baseline evaluation took place in 
1993-1995 with subsequent measurements in 1998-2000, 2003-2005, and 2009-2010. Incident hearing loss was defined as PTA (500, $1,000,2,000$, and $4,000 \mathrm{~Hz}$ ) greater than $25 \mathrm{~dB}$ $\mathrm{HL}$ in either ear. Diabetes was associated with a slightly elevated risk of hearing loss in age- and sex-adjusted models, although this elevated risk was not significant $(\mathrm{HR}=1.26[95 \% \mathrm{CI}$, 0.93-1.71]). However, a greater risk of hearing loss was observed in individuals with poorly controlled diabetes ( $\mathrm{HR}=2.03$ [95\% CI, 1.014.08]). ${ }^{40}$

A longitudinal study of 138,909 women ( $>2.4$ million person-years) from the Nurses' Health Study evaluated the relationship between T2DM and self-reported incident HL. Women with T2M had a significantly higher incidence of (moderate or worse) hearing loss than persons without diabetes in their multivariable-adjusted model (HR $=1.16$ [95\% CI, 1.07-1.27]). ${ }^{41}$

A recent prospective Korean cohort study evaluated 253,301 adults (average age $=37.6$ years $[\mathrm{SD}=7.7]$ ) with normal hearing to determine the association between baseline diabetes and incident hearing loss (defined as PTA [500, $1,000$, and $2,000 \mathrm{~Hz}]$ greater than $25 \mathrm{~dB} \mathrm{HL}$ bilaterally). Individuals were followed up for over 1.3 million person-years (median followup of 4 years) with 2,817 developing hearing loss. There was an increased risk of incident hearing loss in PWD compared with those without diabetes $(\mathrm{HR}=1.36$ [95\% CI, 1.19-1.56]) in a model adjusted for age, sex, study center, noise, body mass, smoking, alcohol, and cardiovascular covariates. $^{42}$

Data are also available from the Blue Mountains Hearing study. Prevalence trends were discussed earlier. Follow-up testing was conducted at the 5-year examination (1997-1999). Interestingly, this study observed a significant association between prevalent DM and hearing loss but not incident DM and hearing loss. Fiveyear progression of hearing loss (defined as $>5$ dB HL increase in PTA [500, 1000, 2000, $4000 \mathrm{~Hz}]$ ) was compared between PWD and those without diabetes. For PWD and normal hearing at baseline, the incidence of moderate hearing loss (defined as PTA $>25 \mathrm{~dB}$ HL) was $18.7 \%$ compared with $18.0 \%$ for persons without diabetes $(\mathrm{OR}=1.01 \quad$ [95\% CI: 0.54-1.91]). Those with newly diagnosed diabetes were more likely to demonstrate progression of hearing loss than participants without diabetes (adjusted OR $=2.71$ [95\% CI, 1.07-6.86]). ${ }^{33}$

The risk of sudden SNHL in PWD has been explored. A retrospective cohort study of individuals in the Taiwan National Health Insurance program, a universal healthcare system that covers $99 \%$ of the country's 23 million residents, compared hearing outcomes between PWD who were receiving treatment and nondiabetics. Sudden SNHL was defined by ICD-9 code (388.2). The sample group was pulled from the Longitudinal Health Insurance Database 2000, which contains a subset of all claims data from 1996 to 2009. Between 2000 and 2004, there were claims data available from 26,556 PWD who met the study criteria. An additional 26,556 nondiabetics were randomly selected. The risk of sudden SNHL was significantly higher in the diabetic group compared with the nondiabetic group in two age groups: $35-$ to 49- and 50- to 64-yearolds. The incident rate ratio for these two age groups were 2.09 (95\% CI, 1.27-3.45) and 1.67 (95\% CI, 1.23-2.26), respectively. Incidence rates were not significantly higher for persons younger than 35 years or $\geq 65$ years. ${ }^{43}$

Recent work has suggested that age-related hearing loss may be a risk factor for dementia. An analysis of data from the Taiwan National Health Insurance Research Database included 4,108 patients with presbycusis and a control cohort (matched by age, sex, and comorbidities) of 4,013 persons without hearing loss. Diabetes was found to be an independent risk factor for incident dementia in persons with hearing loss in multivariable adjusted models. ${ }^{44}$

\section{GESTATIONAL DIABETES AND HEARING LOSS}

Little is known about the association between gestational diabetes and hearing sensitivity. One small case-control study of 27 patients with gestational diabetes and 31 nondiabetic pregnant women found significantly elevated high-frequency auditory thresholds (8,000$14,000 \mathrm{~Hz}$ ) among women with gestational diabetes. ${ }^{45}$ Additional work is needed in this area to better understand the possible relationship between gestational diabetes and auditory damage. 
The influence of gestational diabetes in mothers on hearing sensitivity in their newborns has received limited research attention. Limited data are available, although a retrospective study of 73 infants born to pregestational diabetic women and 73 controls (infants of nondiabetic mothers) does shed some light on this issue. Using DPOAE and ABR screening protocols, a slightly higher referral rate was observed in infants born to diabetic mothers than infants born to nondiabetic mothers (9.4 vs. 5.6\%, respectively). However, referral rates were low $(<10$ cases per group) and the differences were not statistically significant. ${ }^{46}$ At the time of this writing, we were unable to find additional data pertaining to infants born to mothers with gestational diabetes specifically.

\section{DIABETES AND TINNITUS}

Diabetes also may be associated with increased risk of tinnitus, though studies to date are few and inconclusive. A Hungarian study compared self-reported tinnitus prevalence between 103 PWD (mean age $=61.6$ years) and 15,622 persons without diabetes (mean age $=55.1$ years). Hearing loss and/or tinnitus was observed in the majority of PWD (80\%) but in only $14 \%$ of individuals without hearing loss. ${ }^{47}$ Recently, Spankovich et $\mathrm{al}^{48}$ found a relationship between diabetes and tinnitus in data from the NHANES dataset. Participants $(n=8,143)$ aged between 20 and 69 years were considered and DM was significantly correlated with tinnitus.

In contrast, a cross-sectional study of older individuals ( $>60$ years) did not find an association between DM and tinnitus. ${ }^{49}$ Additional population-based data are needed to clarify this relationship.

\section{DIABETES AND MALIGNANT OTITIS EXTERNA}

PWD are more susceptible to infections due to elevated serum glucose levels, which cause an alteration in host immune responses. PWD are particularly more likely to develop malignant otitis externa (MOE). MOE is a rare condition that arises from the external ear canal and can eventually spread to adjacent bone. Studies of MOE have shown that as many as $90 \%$ of patients with this condition are PWD. ${ }^{50}$ Among PWD, the infection is more common in older individuals, those with poor glucose control, and those who wear hearing aids. ${ }^{51}$ The mortality rate from this infection is as high as $50 \%{ }^{52}$

\section{STRENGTH OF THE RELATIONSHIP BETWEEN DIABETES MELLITUS AND HEARING LOSS}

The majority of studies reviewed support a positive association between DM and hearing loss. Studies have identified associations between hearing loss and diabetes in young and middle-aged adults, ${ }^{32,53}$ providing support for the hypothesis that auditory dysfunction related to DM is separate from the pathophysiological changes that accompany aging. It is plausible that the effects of DM on hearing are easier to identify at younger ages before the cumulative effects of aging, noise exposure, ototoxic drugs, lifestyle, and other cardiometabolic risk factors have obscured the relationship.

Interpretation of the combined results of these studies is complicated by several factors. Most, but not all, reports were cross-sectional, preventing conclusions concerning causality. However, incidence data ${ }^{30,38,39}$ lend further support to the causal relationship between diabetes and auditory dysfunction.

Not all studies controlled for potential confounding factors such as socioeconomic status, ototoxic medication, and medical comorbidities. To our knowledge, data are lacking regarding the ability to reverse diabetes-related alterations in hearing, and the relationship between diabetes and tinnitus remains unclear.

Likewise, there is the potential complication of noise exposure, though some investigators have asserted that self-reported noise exposure cannot explain the observed discrepancies in hearing between PWD and those who do not have diabetes $^{32}$ or between PWD employed in lowand high-noise workplaces. ${ }^{54}$ Thus, it appears that aging and noise exposure are only partial explanations for the variance in auditory outcomes observed among PWD and a more critical evaluation of these relationships is warranted.

Last, although the majority of populationbased studies support the connection between DM and hearing loss, data from the 
Epidemiology of Hearing Loss Study, ${ }^{55}$ Framingham Heart Study, ${ }^{56}$ Brazilian Longitudinal Study of Adult Health, ${ }^{36}$ and NHANES ${ }^{57}$ do not.

The lack of significant relationships in these studies may be explained by population characteristics; the Framingham study was limited to older adults (mean age: 73 years), as was the Epidemiology of Hearing Loss Study (mean age: 63 years, although women with DM adjusted for age did show increased odds of hearing loss). ${ }^{55}$ Lin and colleagues ${ }^{57}$ studied 717 adults over the age of 70 years from the 2005-2006 NHANES cycle. They identified a prevalence of hearing loss $(500,1000,2000 \mathrm{~Hz})$ of $16.5 \%$, although this number escalated to $99.7 \%$ when hearing loss was defined as high-frequency PTA (average threshold at 3000, 4000, 6000, and $8000 \mathrm{~Hz}) 15 \mathrm{~dB}$ threshold in the poorer ear was used. However, this study did not observe a significant association between diabetes and hearing loss $(\mathrm{OR}=1.10$ [95\% CI, 0.71-1.70]). Data from the Framingham Heart Study have not demonstrated poorer hearing in elderly PWD compared with persons without diabetes. The Framingham Heart Study is a general population sample identified in 1948. Participants undergo extensive health examinations every 2 years. Audiological examinations were offered at the 18th visit yielding 1,662 elderly subjects. Behavioral thresholds (250$8,000 \mathrm{~Hz}$ ) were obtained and suprathreshold testing, including word recognition and the Synthetic Sentence Identification test, was performed. Neither suprathreshold testing or behavioral thresholds differed significantly between persons with and without diabetes. ${ }^{56}$ Last, the Brazilian Longitudinal Study of Adult Health found a relationship without adjusting for covariates, but did not find a statistically significant relationship when adjusting for age, sex, and hypertension. ${ }^{36}$ Future epidemiological studies examining DM and the relationship with hearing loss should be cognizant of a reduced relationship with age.

\section{SUMMARY}

Sufficient meta-data findings exist to support a conclusion that DM necessitates careful monitoring for complications including those of the visual, renal, and peripheral nervous systems.
Early identification and management of hearing loss is not currently part of ADA guidelines, but its high prevalence among PWD and relationship with evidence of hearing loss at young ages warrants further investigation and possible inclusion in the ADA's recommended comprehensive medical assessment.

\section{CONFLICT OF INTEREST}

The authors have nothing to disclose.

\section{ACKNOWLEDGMENTS}

The authors thank The Audiology Project and Executive Director Dr. Kathy Dowd for ongoing support of this work. Additional gratitude is owed to Drs. Christopher Spankovich and Victor Bray for review of earlier drafts of this manuscript.

\section{REFERENCES}

1. Selvin E, Parrinello CM, Sacks DB, Coresh J. Trends in prevalence and control of diabetes in the United States, 1988-1994 and 1999-2010. Ann Intern Med 2014;160(08):517-525

2. Cowie CC, Rust KF, Ford ES, et al. Full accounting of diabetes and pre-diabetes in the U.S. population in 1988-1994 and 2005-2006. Diabetes Care 2009;32(02):287-294

3. Shaw JE, Sicree RA, Zimmet PZ. Global estimates of the prevalence of diabetes for 2010 and 2030. Diabetes Res Clin Pract 2010;87(01):4-14

4. American Diabetes Association. Diagnosis and classification of diabetes mellitus. Diabetes Care 2004;27(Suppl 1):S5-S10

5. Allen PJ, Vessey JA, Schapiro N. Primary Care of the Child with a Chronic Condition E-Book. Elsevier Health Sciences; 2009

6. Pettitt DJ, Talton J, Dabelea D, et al; SEARCH for Diabetes in Youth Study Group. Prevalence of diabetes in U.S. youth in 2009: the SEARCH for diabetes in youth study. Diabetes Care 2014;37(02):402-408

7. Mølbak AG, Christau B, Marner B, Borch-Johnsen K, Nerup J. Incidence of insulin-dependent diabetes mellitus in age groups over 30 years in Denmark. Diabet Med 1994;11(07):650-655

8. Imperatore G, Boyle JP, Thompson TJ, et al; SEARCH for Diabetes in Youth Study Group. Projections of type 1 and type 2 diabetes burden in the U.S. population aged $<20$ years through 2050: dynamic modeling of incidence, mortality, and population growth. Diabetes Care 2012;35(12): $2515-2520$ 
9. American Diabetes Association. 2. Classification and diagnosis of diabetes. Diabetes Care 2017;40 (Suppl 1):S11-S24

10. Centers for Disease Control and Prevention. National Diabetes Statistics Report: Estimates of Diabetes and Its Burden in the United States, 2014. Atlanta 2014

11. Stokes A, Preston SH. Deaths attributable to diabetes in the United States: comparison of data sources and estimation approaches. PLoS One 2017;12(01):e0170219

12. Donnelly R, Emslie-Smith AM, Gardner ID, Morris $\mathrm{AD}$. $\mathrm{ABC}$ of arterial and venous disease: vascular complications of diabetes. BMJ 2000;320 (7241):1062-1066

13. American Diabetes Association. 3. Comprehensive medical evaluation and assessment of comorbidities. Diabetes Care 2017;40(Suppl 1):S25-S32

14. Jordao AMD. Consideration sur un cas du diabete. Union Medicale du Paris 1857;11:446

15. Malucelli DA, Malucelli FJ, Fonseca VR, et al. Hearing loss prevalence in patients with diabetes mellitus type 1. Rev Bras Otorrinolaringol (Engl Ed) 2012;78(03):105-115

16. Elamin A, Fadlallah M, Tuevmo T. Hearing loss in children with type 1 diabetes. Indian Pediatr 2005; 42(01):15-21

17. Okhovat SA, Moaddab MH, Okhovat SH, et al. Evaluation of hearing loss in juvenile insulin dependent patients with diabetes mellitus. JRes Med Sci 2011;16(02):179-183

18. Hou Y, Xiao X, Ren J, Wang Y, Zhao F. Auditory impairment in young type 1 diabetics. Arch Med Res 2015;46(07):539-545

19. Ferrer JP, Biurrun O, Lorente J, et al. Auditory function in young patients with type 1 diabetes mellitus. Diabetes Res Clin Pract 1991;11(01): $17-22$

20. Botelho CT, Carvalho SA, Silva IN. Increased prevalence of early cochlear damage in young patients with type 1 diabetes detected by distortion product otoacoustic emissions. Int J Audiol 2014; 53(06):402-408

21. Ottaviani F, Dozio N, Neglia CB, Riccio S, Scavini M. Absence of otoacoustic emissions in insulindependent diabetic patients: is there evidence for diabetic cochleopathy? J Diabetes Complications 2002;16(05):338-343

22. Lisowska G, Namysłowski G, Morawski K, Strojek K. Early identification of hearing impairment in patients with type 1 diabetes mellitus. Otol Neurotol 2001;22(03):316-320

23. Teng Z-P, Tian R, Xing F-L, et al. An association of type 1 diabetes mellitus with auditory dysfunction: A systematic review and meta-analysis. Laryngoscope 2017;127(07):1689-1697

24. Zipf G, Chiappa M, Porter KS, Ostchega Y, Lewis BG, Dostal J. National Health and Nutrition
Examination survey: plan and operations, 19992010. Vital Health Stat 1 2013;(56):1-37

25. Cheng YJ, Gregg EW, Saaddine JB, Imperatore G, Zhang X, Albright AL. Three decade change in the prevalence of hearing impairment and its association with diabetes in the United States. Prev Med 2009;49(05):360-364

26. Bainbridge KE, Cheng YJ, Cowie CC. Potential mediators of diabetes-related hearing impairment in the U.S. population: National Health and Nutrition Examination Survey 1999-2004. Diabetes Care 2010;33(04):811-816

27. Bainbridge KE, Hoffman HJ, Cowie CC. Risk factors for hearing impairment among U.S. adults with diabetes: National Health and Nutrition Examination Survey 1999-2004. Diabetes Care 2011;34(07):1540-1545

28. Dalton DS, Cruickshanks KJ, Klein R, Klein BE, Wiley TL. Association of NIDDM and hearing loss. Diabetes Care 1998;21(09):1540-1544

29. Helzner EP, Cauley JA, Pratt SR, et al. Race and sex differences in age-related hearing loss: the Health, Aging and Body Composition Study. J Am Geriatr Soc 2005;53(12):2119-2127

30. Cruickshanks KJ, Dhar S, Dinces E, et al. Hearing impairment prevalence and associated risk factors in the Hispanic Community Health Study/Study of Latinos. JAMA Otolaryngol Head Neck Surg 2015;141(07):641-648

31. Kakarlapudi V, Sawyer R, Staecker H. The effect of diabetes on sensorineural hearing loss. Otol Neurotol 2003;24(03):382-386

32. Austin DF, Konrad-Martin D, Griest S, McMillan GP, McDermott D, Fausti S. Diabetes-related changes in hearing. Laryngoscope 2009;119(09): 1788-1796

33. Mitchell P, Gopinath B, McMahon CM, et al. Relationship of Type 2 diabetes to the prevalence, incidence and progression of age-related hearing loss. Diabet Med 2009;26(05):483-488

34. Uchida Y, Sugiura S, Ando F, Nakashima T, Shimokata H. Diabetes reduces auditory sensitivity in middle-aged listeners more than in elderly listeners: a population-based study of age-related hearing loss. Med Sci Monit 2010;16(07):PH63-PH68

35. Sakuta H, Suzuki T, Yasuda H, Ito T. Type 2 diabetes and hearing loss in personnel of the SelfDefense Forces. Diabetes Res Clin Pract 2007;75 (02):229-234

36. Samelli AG, Santos IS, Moreira RR, et al. Diabetes mellitus and sensorineural hearing loss: is there an association? Baseline of the Brazilian Longitudinal Study of Adult Health (ELSA-Brasil). Clinics (São Paulo) 2017;72(01):5-10

37. Mozaffari M, Tajik A, Ariaei N, Ali-Ehyaii F, Behnam H. Diabetes mellitus and sensorineural hearing loss among non-elderly people. East Mediterr Health J 2010;16(09):947-952 
38. Akinpelu OV, Mujica-Mota M, Daniel SJ. Is type 2 diabetes mellitus associated with alterations in hearing? A systematic review and meta-analysis. Laryngoscope 2014;124(03):767-776

39. Horikawa C, Kodama S, Tanaka S, et al. Diabetes and risk of hearing impairment in adults: a metaanalysis. JClin Endocrinol Metab 2013;98(01): $51-58$

40. Cruickshanks KJ, Nondahl DM, Dalton DS, et al. Smoking, central adiposity, and poor glycemic control increase risk of hearing impairment. J Am Geriatr Soc 2015;63(05):918-924

41. Gupta S, Eavey RD, Wang M, Curhan SG, Curhan GC. Type 2 diabetes and the risk of incident hearing loss. Diabetologia 2019;62(02): 281-285

42. Kim MB, Zhang Y, Chang Y, et al. Diabetes mellitus and the incidence of hearing loss: a cohort study. Int J Epidemiol 2017;46(02):717-726

43. Lin SW, Lin YS, Weng SF, Chou CW. Risk of developing sudden sensorineural hearing loss in diabetic patients: a population-based cohort study. Otol Neurotol 2012;33(09):1482-1488

44. Su P, Hsu CC, Lin HC, et al. Age-related hearing loss and dementia: a 10-year national populationbased study. Eur Arch Otorhinolaryngol 2017;274 (05):2327-2334

45. Selcuk A, Terzi H, Turkay U, Kale A, Genc S. Does gestational diabetes result in cochlear damage? J Laryngol Otol 2014;128(11):961-965

46. Stanton SG, Ryerson E, Moore SL, SullivanMahoney M, Couch SC. Hearing screening outcomes in infants of pregestational diabetic mothers. Am J Audiol 2005;14(01):86-93

47. Somogyi A, Rosta K, Vaszi T. Hearing impairment and tinnitus in patients with type 2 diabetes. Orv Hetil 2013;154(10):363-368
48. Spankovich C, Bishop C, Johnson MF, et al. Relationship between dietary quality, tinnitus and hearing level: data from the national health and nutrition examination survey, 1999-2002. Int J Audiol 2017;56(10):716-722

49. Gibbin KP, Davis CG. A hearing survey in diabetes mellitus. Clin Otolaryngol Allied Sci 1981;6(05): 345-350

50. Rubin Grandis J, Branstetter BF IV, Yu VL. The changing face of malignant (necrotising) external otitis: clinical, radiological, and anatomic correlations. Lancet Infect Dis 2004;4(01):34-39

51. Carfrae MJ, Kesser BW. Malignant otitis externa. Otolaryngol Clin North Am 2008;41(03):537-549, viii-ix

52. Deresinski S. Infections in the diabetic patient: strategies for the clinician. Infect Dis Rep 1995;1:1-12

53. Bainbridge KE, Hoffman HJ, Cowie CC. Diabetes and hearing impairment in the United States: audiometric evidence from the National Health and Nutrition Examination Survey, 1999 to 2004. Ann Intern Med 2008;149(01):1-10

54. Hodgson MJ, Talbott E, Helmkamp JC, Kuller LH. Diabetes, noise exposure, and hearing loss. J Occup Med 1987;29(07):576-579

55. Zhan W, Cruickshanks KJ, Klein BEK, et al. Modifiable determinants of hearing impairment in adults. Prev Med 2011;53(4-5):338-342

56. Gates GA, Cobb JL, D'Agostino RB, Wolf PA. The relation of hearing in the elderly to the presence of cardiovascular disease and cardiovascular risk factors. Arch Otolaryngol Head Neck Surg 1993;119(02):156-161

57. Lin FR, Thorpe R, Gordon-Salant S, Ferrucci L. Hearing loss prevalence and risk factors among older adults in the United States. J Gerontol A Biol Sci Med Sci 2011;66(05):582-590 